\title{
POSICIONAMENTO POR PONTO DE ALTA PRECISÃO UTILIZANDO O GPS: UMA SOLUÇÃO PARA A GEODINÂMICA
}

\author{
J. F. G. Monico
}

Posicionamento por ponto a partir de dados GPS pode proporcionar precisão que varia de 100 metros, até poucos milímetros, ao nível de probabilidade de $95 \%$. Para alcançar o melhor nível de precisão, o usuário deve dispor de equipamento e programas de computador adequados, bem como capacidade para acessar produtos GPS disponibilizados pelo International GPS Geodynamics Service. Neste trabalho descreve-se a teoria do posicionamento por ponto com o GPS e apresentam-se os resultados de um experimento realizado utilizando dados da Rede Brasileira de Monitoramento Contínuo dos satélites GPS. Os resultados proporcionaram repetibilidade melhor que $5 \mathrm{~mm}$ e $10 \mathrm{~mm}$ para as componentes horizontais $\mathrm{N}$ e E respectivamente, e de $6 \mathrm{~mm}+4$ ppb (partes por bilhão) para a componente vertical (h). Comparação com resultados estimados na realização do SIRGAS mostrou-se compatível com a incerteza das estações que foram utilizadas para conectá-lo ao ITRF94. Trata-se portando de um método com grande potencialidade de ser utilizado em aplicações requerendo alta precisão, como por exemplo algumas aplicações em Geodinâmica.

Palavras-chave: Posicionamento GPS de alta precisão; Correção dos relógios dos satélites; Repetibilidade

POINT POSITIONING OF HIGH ACCURACY USING THE GPS- A SOLUTION FOR GEODYNAMICS - Point positioning from GPS data can provide precision varying from 100 meters to a few millimeters at the level of 95\% probability. To reach the best level of accuracy, users need proper equipment and software, as well as access capability to GPS products available at the International GPS Geodynamics Service. In this paper, the theory related to point positioning using GPS is presented as well as the results of an experiment conducted using data from the Brazilian Active Control System. The results show repeatability better than $5 \mathrm{~mm}$ and $10 \mathrm{~mm}$ for the $N$ and $E$ baseline components respectively, and $6 \mathrm{~mm}+4 \mathrm{ppb}$ (parts per billion) for the vertical. Comparison with SIRGAS campaign showed results at the same level of uncertainty as that of the stations used to tie the SIRGAS frame to ITRF94. Therefore, precise point positioning is a powerful tool to be used in applications requiring high level of precision, such as Geodynamics.

Key words: GPS Positioning; High Precision Positioning; Satellite Clock Corrections; Repeatability.

Departamento de Cartografia, FCT/UNESP

Rua Roberto Simonsen 305, CEP 19060-900

Presidente Prudente, SP, Brasil, fone: 0182221575

e-mail: galera@prudente.unesp.br 


\section{INTRODUÇÃO}

No posicionamento com GPS (Global Positioning System), o termo posicionamento por ponto normalmente refere-se a obtenção da posição de uma estação a partir de observações de pseudo-distâncias, derivadas do código C/A (Clear/Acquisition), e fixando a órbita e demais parâmetros dos satélites aos valores contidos nas mensagens de navegação (broadcast ephemeris). Trata-se do serviço proporcionado pelo GPS sob a denominação de SPS (Standard Positioning Service). Neste caso, o posicionamento instantâneo de uma estação apresenta precisão planimétrica e altimétrica da ordem de 100 e $140 \mathrm{~m}$, respectivamente, com probabilidade de $95 \%$. Se o receptor permanecer estacionário sobre a estação, coletando observações por um longo período de tempo, a qualidade dos resultados não melhora consideravelmente, face ao fato dos dados envolvidos no processamento conterem erros de natureza sistemática. Caso esteja disponível, é possível incluir no processamento, além da pseudo-distância, a medida da fase onda portadora. No entanto, tal procedimento não tem sido uma prática comum neste tipo de posicionamento, pois não proporciona considerável refinamento da solução. Desta forma, esta técnica não atende os requisitos de precisão intrínseco ao posicionamento geodésico. No contexto deste trabalho, esta técnica será denominada posicionamento por ponto convencional.

Os principais erros afetando esta técnica estão relacionados com a qualidade (precisão) da observável utilizada (pseudo-distância) e a acuracidade dos parâmetros transmitidos nas mensagens de navegação. No que concerne ao último, enquanto a acuracidade da órbita do satélite é da ordem de poucos metros, a dos relógios dos satélites é uma ordem de magnitude maior (Zumberge \& Bertiger, 1996). Acrescentam-se à estes erros aqueles advindos da refração troposférica e ionosférica, multicaminho do sinal, dentre outros (Monico, 1998).

Para os casos em que não há necessidade de posicionamento em tempo real, ou seja, as observações podem ser pós-processadas, é possível utilizar as efemérides precisas e as correções para os relógios dos satélites produzidos pelo International GPS Geodynamics Service (IGS) (Bock, 1996), ambos com precisão de poucos centímetros. Estes produtos podem ser utilizados no processamento de observações de pseudo-distâncias, fase da onda portadora, ou ambas, as quais podem ter sido coletadas por receptores de uma ou duas frequiências. O procedimento em que se utiliza as observações de pseudo-distâncias, quer sejam de receptores de uma ou duas freqüências, tem sido utilizado a algum tempo pelo Sistema de Controle Ativo Canadense (Canadian Active Control System) do Natural Resources of Canada (NRCAn), que é um dos colaboradores do IGS. Resultados têm mostrado precisão da ordem de um metro no posicionamento 3$\mathrm{D}$, empregando-se uma única época de observação (Héroux \& Kouba, 1995). Este procedimento será denominado neste trabalho de posicionamento por ponto preciso, o qual pode ser aplicado numa grande variedade de atividades.

Utilizando as observáveis fase da onda portadora e pseudo-distâncias coletadas por receptores de dupla freqüência, também em conjunto com os produtos IGS, o posicionamento por ponto pode proporcionar resultados similares aos casos em que dados de vários receptores são processados em conjunto, numa rede GPS. E o que é mais importante, com um baixo custo computacional (Zumberge et al., 1997a). Este método será denominado neste trabalho de posicionamento por ponto de alta precisão. Vale no entanto ressaltar que se trata de um método que não se aplica ao posicionamento em tempo real.

Neste trabalho será apresentada a fundamentação teórica dos três tipos de posicionamento por ponto citados anteriormente, com ênfase no posicionamento de alta precisão. Trata-se do caso que apresenta grande potencialidade de ser utilizado em aplicações de geodinâmica, com grandes vantagens sobre o processamento de redes GPS, onde há grande dispêndio computacional. Visando mostrar a precisão e acuracidade que podem ser obtidos com esta técnica, o processamento de uma amostra de dados das estações pertencentes a Rede Brasileira de Monitoramento Contínuo do Satélites GPS (RBMC), estabelecida pelo IBGE (Fundação Instituto Brasileiro de Geografia e Estatística) (Fortes, 1997) será apresentado, bem como a análise dos resultados. $\mathrm{O}$ processamento e análise serão precedidos de uma breve descrição do software utilizado no trabalho. Trata-se do programa GIPSYOASIS II (GOA-II), desenvolvido no JPL (Jet Propulsion Laboratory).

\section{POSICIONAMENTO POR PONTO CONVENCIONAL}

Considere um receptor A, coletando pseudodistâncias dos satélites visíveis num instante qualquer. Cada observação coletada gera uma equação que comporá o sistema de equações. A equação de observação linearizada é dada por (Monico, 1998): 


$$
\begin{aligned}
E\left(\Delta P D_{A}^{j}\right)= & a_{A}^{j} \Delta X_{A}+b_{A}^{j} \Delta Y_{A}+c_{A}^{j} \Delta Z_{A}+ \\
& +c\left(d t_{A}-d t^{j}\right)+I_{A}^{j}+T_{A}^{j}
\end{aligned}
$$

com $E\left(\triangle P D_{A}^{j}\right)$ sendo a esperança matemática da diferença entre a pseudo-distância observada entre a estação A e o satélite $j\left(P D_{A}^{j}\right)$ e a calculada em função dos parâmetros aproximados $\left(\rho_{A 0}^{j}\right)$. Os coeficientes $a_{A}^{j}, b_{A}^{j} e c_{A}^{j}$ são obtidos a partir da linearização da equação de observação. Tratam-se dos elementos que compõem a matriz A do ajustamento pelo método paramétrico (Gemael, 1994). O fator c é a velocidade da luz no vácuo. Trata-se de uma equação com 4 incógnitas $\left(\Delta \mathrm{X}_{\mathrm{A}}, \Delta \mathrm{Y}_{\mathrm{A}}, \Delta \mathrm{Z}_{\mathrm{A}}\right.$ e dt $\left.\mathrm{A}\right)$, relativos as três correções às coordenadas da estação e o erro do relógio do receptor, respectivamente. Assim sendo, exige-se a presença de no mínimo quatro satélites para obter uma posição instantânea (tempo real). O erro do relógio do satélite $\left(\mathrm{dt}^{\mathrm{j}}\right)$ pode ser calculado a partir das informações contidas nas efemérides dos satélites, as quais estão eivadas dos efeitos da SA (Selective Availability - Disponibilidade Seletiva), um processo intencional de degradação da capacidade de posicionamento com o código C/A (Course Acquisition) (Leick, 1995). A refração troposférica (ionosfera $\left(I_{A}^{j}\right)$ e troposfera $\left(T_{A}^{j}\right)$ ) é normalmente negligenciada. Quando apenas 3 satélites são visíveis, pode-se obter uma posição bi - dimensional. Mas se o receptor estiver em repouso, pode-se processar os dados num ajustamento seqüencial, sem a necessidade da presença de quatro satélites para obtenção de uma posição tri - dimensional.

Nesta técnica de posicionamento, as coordenadas da estação ficarão influenciadas pela SA, através das coordenadas e correções dos relógios dos satélites, além de outros erros, tais como os advindos da refração troposférica, limitando sua precisão àquela estipulada pelo GPS para o SPS.

\section{POSICIONAMENTO POR PONTO PRECISO}

No posicionamento por ponto preciso, a equação de observação utilizada é também aquela expressa pela equação (1), podendo-se dispor da mesma para as duas portadoras $\left(\mathrm{L}_{1}\right.$ e $\left.\mathrm{L}_{2}\right)$, caso em que se pode reduzir consideravelmente os efeitos da refração ionosférica, a partir do uso de combinação linear adequada. Para usuários com receptores de apenas uma frequiência, há duas possibilidades no que se refere a refração ionosférica: negligenciá-la, ou utilizar algum modelo disponível. E vale ressaltar que desde maio de 1998, o IGS está produzindo um modelo global para a ionosfera. Além disto, estudo de modelos para aplicações regionais estão em desenvolvimento no Brasil (Camargo, 1999). No que se refere a refração troposférica, alguns dos vários modelos disponíveis (Silva, 1998) podem ser utilizados.

O ponto central desta técnica é a não utilização das efemérides e correções dos relógios dos satélites contidos na mensagem de navegação, as quais estão degradadas pelos efeitos da SA. Desta forma, estes parâmetros devem ser disponibilizados para os usuários por alguma fonte independente.

Atualmente, o IGS produz três tipos de efemérides e correções para o relógio dos satélites:

- IGS, a qual resulta da combinação das órbitas produzidas pelos centros de análises do IGS, e fica disponível com um período de 7 a 10 dias de atraso,

- IGR, resultante da combinação das órbitas rápidas produzidas pelos centros de análise, ficando disponível com 24 horas de atraso e

- IGP, que se trata das órbitas preditas, disponível com uma hora de antecedência do seu

\begin{tabular}{lllllllll}
\hline & & \multicolumn{1}{c}{ Sat } & Ano & Mês & Dia & H & m & s \\
\hline C & Emr & PrnO6 & 1994 & 06 & 11 & 00 & 00 & .0000 \\
\hline C & Emr & Prno4 & 1994 & 06 & 11 & 00 & 00 & .0000 \\
& & $\ldots$ & & & & & & \\
C & Emr & Prn22 & 1994 & 06 & 11 & 00 & 00 & .0000 \\
C & Emr & Prno6 & 1994 & 06 & 11 & 00 & 00 & 30.000 \\
C & Emr & PrnO4 & 1994 & 06 & 11 & 00 & 00 & 30.000 \\
& $\cdots$ & & & & & & & \\
C & Emr & Prn22 & 1994 & 06 & 11 & 00 & 00 & 30.000 \\
\hline
\end{tabular}

Tabela 1- Exemplo de correções dos relógios dos satélites produzido pelo GSD.

Table 1- Example of satellite clock correction produced by GSD. 
período de validade.

Estes produtos podem ser acessados na Internet, via anonymous ftp, em vários sites; dentre eles, podese citar: http://www.cddisa.gsfc.nasa.gov.

No que diz respeito a qualidade das efemérides, elas apresentam precisão da ordem de 0,10,0,50 e 1 m para as órbitas IGS, IGR e IGP respectivamente. As correções para os relógios dos satélites, pelo menos para os produtos denominados IGS, apresentam precisão da ordem de poucos centímetros. Estes produtos são disponibilizadas com espaçamento de 15 minutos. Este intervalo é adequado para realizar interpolações das órbitas dos satélites, mas não para as correções dos relógios dos mesmos, face a alta variabilidade devido aos efeitos da SA, o que acarreta uma grande degradação na qualidade das correções interpoladas.

Visando reduzir a degradação resultante da interpolação, o Geodetic Survey Division (GSD), do Natural Resources of Canada (NRCan) gera, além das órbitas e correções dos relógios dos satélites GPS no formato padrão do IGS ( com intervalo de 15 minutos), as correções dos relógios dos satélites, utilizando dados CACS (Canadian Active Control System), com intervalo de 30 segundos (Heroux \& Kouba, 1995). A Tab. 1 mostra um trecho de um arquivo de correção dos relógios dos satélites produzido pelo GDS, que é identificado pelo seu antigo nome, EMR.

Trabalho similar vem sendo realizado pelo JPL. Eles têm produzido órbita e correções para os relógios dos satélites, no formato SP3, com intervalo de 30 segundos. Estes produtos podem ser acessados na Internet via anonymous ftp em <sideshow.jpl.nasa.gov>.

As correções para os relógios dos satélites, em conjunto com as efemérides precisas, podem proporcionar posicionamento por ponto, utilizando apenas pseudo-distâncias de uma única época de observação, com precisão da ordem de $1 \mathrm{~m}$ ou melhor.

\section{POSICIONAMENTO POR PONTO DE ALTA PRECISÃO}

No posicionamento por ponto de alta precisão, é imprescindível ter a disposição dados de receptores de dupla frequiência, isto é, pseudo-distância e fase da onda portadora nas duas portadoras. Desta forma, a equação (1) pode ser escrita para as pseudodistâncias em $\mathrm{L}_{1}$ e $\mathrm{L}_{2}$. Além disto, duas equações de fase da onda portadora também fazem parte do processamento. As equações de observação linearizadas são dadas por (Monico, 1998):

$$
\begin{aligned}
& E\left(\lambda_{1} \Delta \phi{ }_{\mathrm{A} 1}^{\mathrm{j}}\right)=a_{A}^{j} \Delta X_{A}+b_{A}^{j} \Delta Y_{A}+c_{A}^{j} \Delta Z_{A}+ \\
& \quad+\mathrm{c}\left(\mathrm{dt}_{\mathrm{A}}-\mathrm{dt}^{\mathrm{j}}\right)+\mathrm{T}_{\mathrm{A}}^{\mathrm{j}}-I_{A}^{j}+\lambda_{1}\left[\phi^{j}\left(t_{0}\right)_{1}-\right. \\
& \left.\quad-\phi_{\mathrm{A}}\left(t_{0}\right)_{1}+N_{A 1}^{j}\right] \\
& E\left(\lambda_{2} \Delta \phi_{\mathrm{A} 2}^{\mathrm{j}}\right)=a_{A}^{j} \Delta X_{A}+b_{A}^{j} \Delta Y_{A}+c_{A}^{j} \Delta Z_{A}+ \\
& \quad+\mathrm{c}\left(\mathrm{dt}_{\mathrm{A}}-\mathrm{dt}^{\mathrm{j}}\right)+\mathrm{T}_{\mathrm{A}}^{\mathrm{j}}-\bar{I}_{A}^{j}+\lambda_{2}\left[\phi^{j}\left(t_{0}\right)_{2}-\right. \\
& \left.\quad-\phi_{\mathrm{A}}\left(t_{0}\right)_{2}+N_{A 2}^{j}\right]
\end{aligned}
$$

Nesta equação tem-se, além dos elementos já apresentados, os seguintes:

$\Delta \phi_{\mathrm{A} 1}^{\mathrm{j}}$ e $\Delta \phi_{\mathrm{A} 2}^{\mathrm{j}}$ referem-se as fases das ondas portadoras observadas e subtraídas das calculadas em função dos parâmetros aproximados para $\mathrm{L}_{1}$ e $\mathrm{L}_{2}$, respectivamente;

$\lambda_{1}$ e $\lambda_{2}$ são os comprimentos de onda das portadoras $\mathrm{L}_{1} \mathrm{e} \mathrm{L}_{2}$, respectivamente;

$\bar{I}_{A}^{j}$ é a refração ionosférica na portadora $\mathrm{L}_{2}$ para o satélite $\mathrm{j}$;

$\phi^{j}\left(t_{0}\right)_{1}$ e $\phi^{j}\left(t_{0}\right)_{2}$ referem-se, respectivamente, às fase das portadoras $\mathrm{L}_{1} \mathrm{e} \mathrm{L}_{2}$, geradas no satélite $\mathrm{j}$, para uma época de referência $t_{0}$;

$\phi_{A}\left(t_{0}\right)_{1}$ e $\phi_{A}\left(t_{0}\right)_{2}$ referem-se, respectivamente, às fase das portadoras $\mathrm{L}_{1} \mathrm{e} \mathrm{L}_{2}$, geradas no receptor $\mathrm{A}$, para uma época de referência $\mathrm{t}_{0} \mathrm{e}$;

$N_{A_{1}}^{j}$ e $N_{A_{2}}^{j}$ representam as ambigüidades nas portadoras $\mathrm{L}_{1}$ e $\mathrm{L}_{2}$, respectivamente.

Trata-se, portanto, de um processamento que envolve quatro observáveis para cada um dos satélites visíveis em cada época. As duas observáveis de fase de batimento da onda portadora podem ser combinadas linearmente, reduzindo sobremaneira os efeitos da refração ionosférica. Procedimento similar pode ser realizado com as pseudo-distâncias. A utilização de um dos vários modelos disponíveis para modelar os efeitos da refração troposférica, em conjunto com alguma técnica de parametrização, reduz os efeitos da refração troposférica. Conforme já citado, pode-se ainda utilizar as efemérides produzidas pelo IGS, ficando o posicionamento isento dos efeitos da SA.

Maiores detalhes sobre o procedimento apresentado neste tópico podem ser encontrados em Zumberge et al. (1997a), onde foi mostrado que com esta técnica é possível obter precisão de poucos milímetros e de poucos centímetros para as 
componentes horizontal e vertical, respectivamente. Neste caso, trata-se do posicionamento por ponto estático, para um período de 24 horas de dados, coletados a uma taxa de 30 segundos. Embora não esteja explícito no texto, o intervalo entre os dados utilizados no processamento foi, aparentemente, de 5 $\min$.

Numa outra experiência, Zumberge et al. (1997b) descreve uma aplicação de posicionamento por ponto de alta precisão, para um receptor em movimento, coletando dados a uma taxa de 5 segundos. Para este caso, numa etapa anterior, os erros dos relógios dos satélites foram estimados a cada 30 segundos. Resultados mostraram que cada posição do receptor pode ser obtida com precisão da ordem de $7 \mathrm{~cm}$.

\section{DADOS ENVOLVIDOS NO EXPERIMENTO}

Visando avaliar o desempenho do posicionamento por ponto de alta precisão, vários processamentos foram realizados para este fim. Os dados foram disponibilizados pelo IBGE, os quais foram coletados pelas estações da RBMC, a qual é composta atualmente por 10 (dez) estações. A Fig. 1 ilustra a distribuição das estações da RBMC (Fortes, 1997).

A Tab. 2 mostra as estações com dados envolvidos no processamento. A não inclusão de dados de algumas estações se deve ao fato dos mesmos não estarem disponíveis, devido a algum tipo de problema durante o período utilizado no processamento.

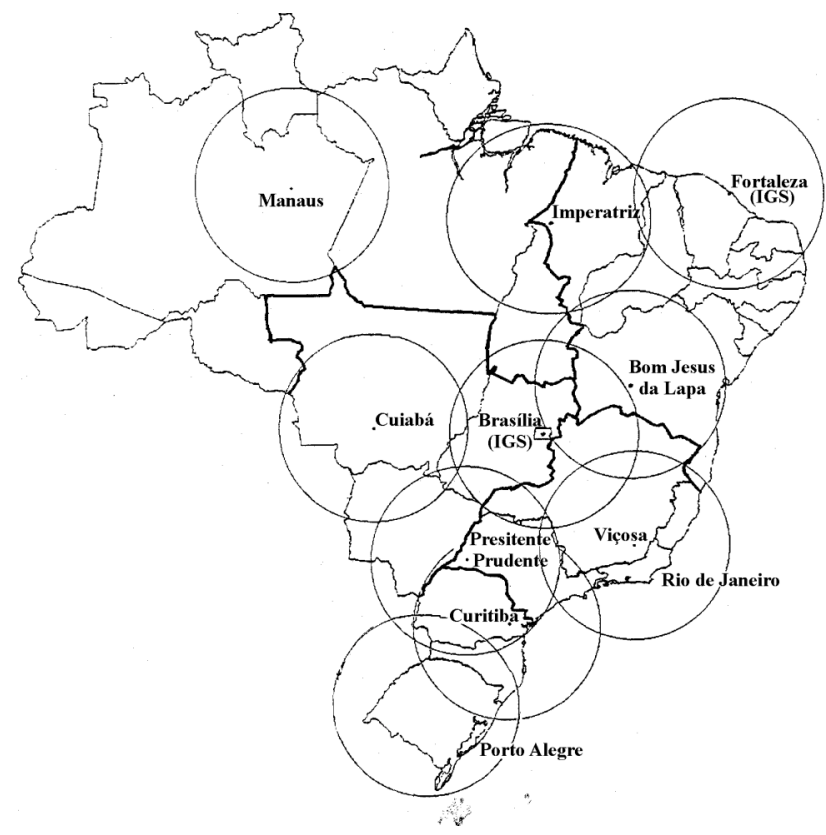

Figura 1- Distribuição das estações da RBMC.

Figure 1- Distribution of the RBMC stations.

\begin{tabular}{|c|c|c|c|c|c|c|c|c|c|c|c|c|c|c|c|c|c|c|c|}
\hline & \multicolumn{19}{|c|}{ Dia do Ano (1998) } \\
\hline Estăcōes & 91 & & \begin{tabular}{l|l|}
3 & 94 \\
\end{tabular} & 9696 & & 21 & 122 & 123 & 124 & 125 & 126 & 127 & 152 & 153 & 154 & 155 & 156 & & 158 \\
\hline BOMJ & $x x$ & & $x \mid x x$ & $x x \mid x x$ & & $x x$ & $x \mathrm{xx}$ & $x x$ & $x x$ & $x x$ & $x x$ & $x x$ & $x x$ & XX & $x x$ & $x X$ & & $x \mathrm{x}$ & $x X$ \\
\hline BRAZ & & & & & & $x x$ & $x x$ & $x x$ & $x x$ & $x x$ & XX & $x x$ & & & & & & & \\
\hline CUIB & $x X$ & $x x \mid x$ & $x \mid x x$ & & & $\overline{x x}$ & $x x$ & $x x$ & $x x$ & $x x$ & $x x$ & $x x$ & $x x$ & $x x$ & $x x$ & $x x$ & XX & \begin{tabular}{l|l}
$x x$ & $\gamma$ \\
\end{tabular} & XX \\
\hline FORT & $X X$ & $\overline{x x} \mid x$ & $x \mid x x$ & & & $\mathrm{XX}$ & $x x$ & $x x$ & $x X$ & $x x$ & $x x$ & $x x$ & XX & $x x$ & $x x$ & $x x$ & XX & $x x+1$ & $x x$ \\
\hline IMPZ & $x X$ & $\overline{x x} x$ & $x|x x|$ & & & $X X$ & $x x$ & $x x$ & $x X$ & $x x$ & $x x$ & $x x$ & & & & & & & \\
\hline MANA & $x x$ & $x \mid x$ & & & & $x x$ & $x x$ & $x x$ & $x x$ & $x x$ & $x x$ & $x x$ & $x X$ & $x x$ & $x x$ & $x \mathrm{x}$ & $x x$ & \begin{tabular}{l|l}
$x x$ & 1 \\
\end{tabular} & XX \\
\hline PARA & $x x$ & $x x \mid x$ & $x \mid x x$ & $x x \mid x x$ & & & & & & & & & $x X$ & $x x$ & $x x$ & $x x$ & $x x$ & \begin{tabular}{l|l}
$x x$ & 1 \\
\end{tabular} & $x x$ \\
\hline UEPP & $x x$ & $x x x$ & $\overline{x x X}$ & $x x \mid x x$ & & $x x$ & $x x$ & $x x$ & $x x$ & XX & $x x$ & $x x$ & $x x$ & $x x$ & $x x$ & $x x$ & $x x$ & $x x+1$ & $x x$ \\
\hline VICO & $x x$ & & & & $x y$ & $\overline{X X}$ & $x X$ & $\mathrm{xx}$ & $x x$ & $x \mathrm{xx}$ & $X X$ & $x x$ & $X X$ & $x x$ & $x x$ & $x x$ & $x x$ & $\begin{array}{lll}x & 1 \\
\end{array}$ & $x X$ \\
\hline
\end{tabular}

Tabela 2- Estações com dados utilizados no processamento em 1998

Table 2- Data from stations used in the processing during 1998.

PROGRAMA DE COMPUTADOR UTILIZADO NO PROCESSAMENTO

Os dados referente as informações que constam da Tab. 2 foram processados utilizando o programa GIPSY-OASIS II (GOA-II), propício para realizar vários tipos de processamentos; incluindo dados do GPS, SLR (Satellite Laser Range) e DORIS (Doppler Orbitography and Radio Positioning Integrated by Satellite). No caso do GPS, os dados de entrada devem estar no formato RINEX (Receiver Independent EXchange format) (Gurtner, 1994). O ambiente computacional utilizado foi uma estação de trabalho Sun Ultra 1 com sistema operacional SunOS 5.5.1.

O programa GOA-II foi desenvolvido pelo JPL e permite a realização de posicionamento por ponto de alta precisão. Além desta, várias outras opções fazem parte deste sistema, que pode ser considerado um dos programas que representa o estado da arte em processamento de dados GPS. Maiores detalhes sobre o GOA-II podem ser encontrados em Webb \& Zumberge (1997). No posicionamento por ponto de alta precisão, as duas observáveis são utilizadas, as quais devem estar disponíveis nas duas portadoras. Isto permite reduzir os efeitos da ionosfera a partir do uso de combinação linear apropriada. Pode-se utilizar efemérides precisas produzidas pelo IGS, ou pelo próprio JPL, bem como as efemérides transmitidas ou as produzidas pelo GSD, em conjunto com as correções dos relógios. No primeiro caso, como as correções dos relógios são produzidas apenas a cada 15 minutos, somente observações coletadas nos instantes coincidentes com as correções fazem parte do processamento, o que reduz consideravelmente o número de observações passíveis de serem incluídas no processamento. Utilizando as efemérides produzidas 


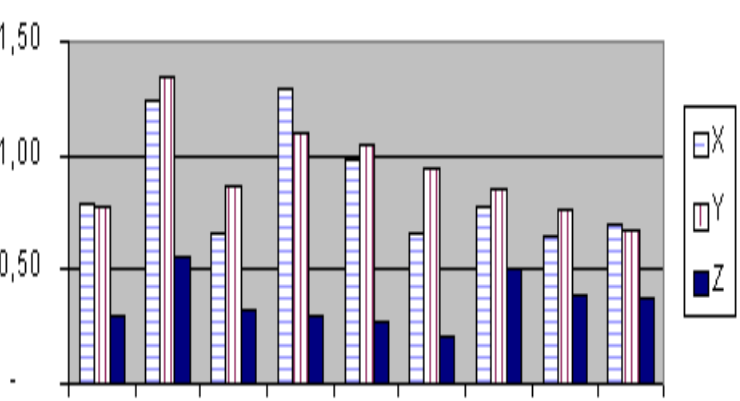

BOMJ BRAZ CUIB FORT MPZ MANA PARA UEPP VICO Estaçōes

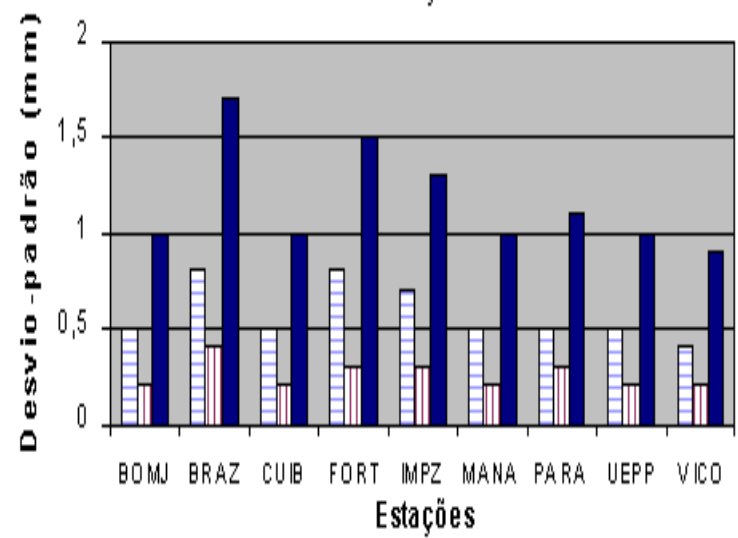

Figura 2- Desvio-padrão das coordenadas das estações processadas.

Figure 2- Coordinates' standard deviation of the processed stations.

pelo JPL, próprias para serem utilizadas com o programa GIPSY, todas as observáveis podem ser utilizadas. Os produtos produzidos pelo GSD seria uma outra opção, mas eles não estão em formato apropriado para serem utilizados com o programa GOA-II.

\section{ESTRATÉGIA APLICADA NA ESTIMATI- VA DOS PARÂMETROS}

No posicionamento por ponto de alta precisão, fizeram parte do processamento as observações referentes a fase da onda portadora e pseudo-distância, disponíveis em L1 e L2. A observável básica foi a combinação linear livre dos efeitos da ionosfera (ionospheric free observable), denominada L3. Adotou-se 30 segundos como o intervalo de processamento dos dados, com ângulo de elevação igual ou superior a 150. No que se refere a troposfera, o resíduo do atraso troposférico zenital foi tratado estocásticamente, num processo denominado random walk process (Bierman, 1977). Os erros dos relógios dos receptores foram estimados no processamento, adotando-se como oscilador padrão o da estação FORT.

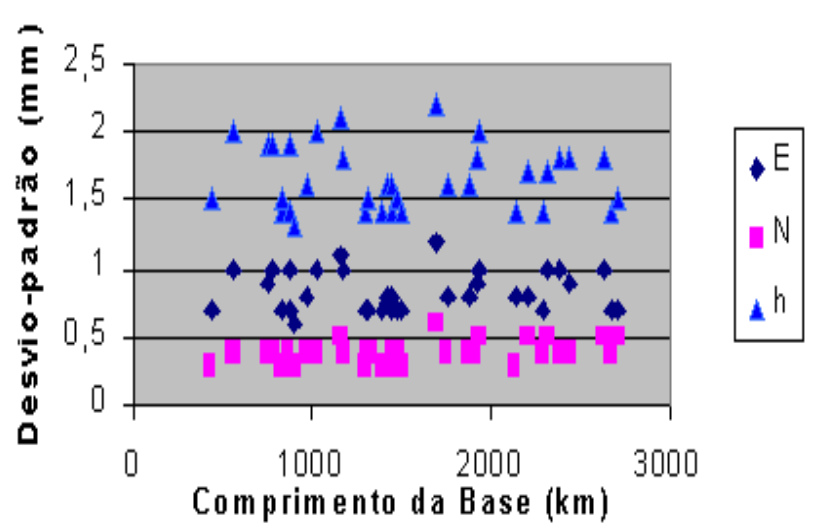

Figura 3- Desvio-padrão das componentes das linhas bases. ,

Figure 3- Standard deviation of the baseline components.

As órbitas e correções dos relógios dos satélites utilizados no processamento foram os produzidos pelo JPL, acessível via ftp anonymous (sideshow.jpl.nasa.gov). Eles são produzidos num ajustamento livre e as estações envolvidas transformadas posteriormente para o ITRF96 (Boucher et al., 1997). Desta forma, os resultados obtidos no processamento foram também transformados para este referencial, mediante a aplicação dos parâmetros fornecidos pelo próprio JPL.

\section{ANÁLISE DOS RESULTADOS}

A análise da precisão e acuracidade em posicionamento geodésico tem sido, em geral, baseada em três métodos:

- cálculo do desvio-padrão formal, baseado na geometria envolvida no processamento (matriz A) e matriz variância-covariância (MVC) das observações (precisão);

- repetibilidade das coordenadas estimadas (precisão) e

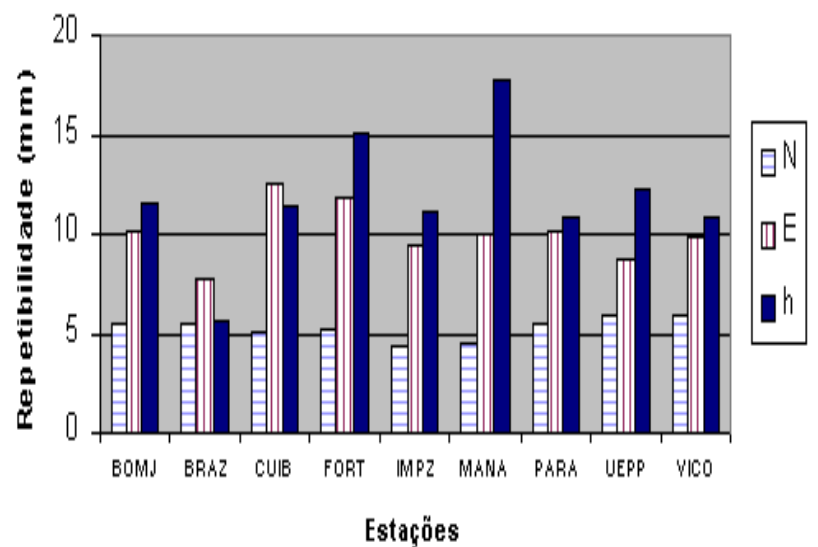

Figura 4- Repetibilidade das coordenadas das estações.

Figure 4- Repeatability of the station coordinates. 

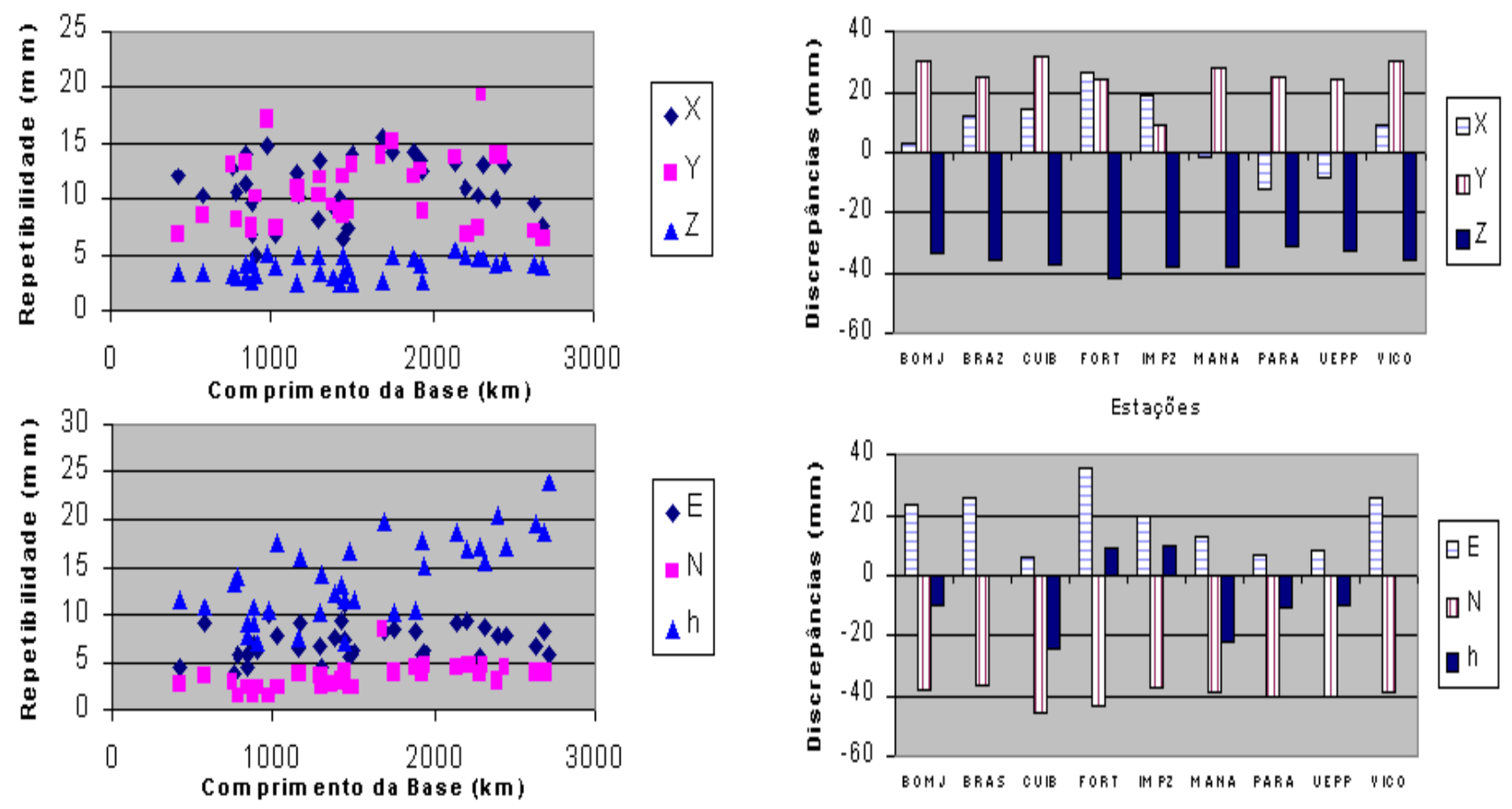

Figura 5- Repetibilidade das componentes das linhas bases

Figure 5- Repeatability of the baseline components

- comparação com outras técnicas e processamentos (acuracidade).

O desvio padrão formal dos parâmetros é obtido a partir da MVC dos parâmetros, obtida no processamento a partir de técnica de Filtro de Kalman. Trata-se de uma expressão que normalmente proporciona valores otimistas para a qualidade dos parâmetros. A Fig. 2 ilustra a precisão formal das coordenadas Este (E), Norte $(\mathrm{N})$ e altitude geométrica (h), bem como nas coordenadas cartesianas X, Y e Z, para as estações envolvidas no processamento.

A estação BRAZ, que apresentou os piores resultados, é a que teve menor número de dias de dados envolvidos no processamento (ver Tab.1). Pode-se também observar que, em termos de coordenadas cartesianas, a componente $\mathrm{Z}$ é aquela que proporciona os melhores resultados. Entretanto, é relevante notar que a altitude geométrica (h) é da ordem de duas vezes pior que as componentes horizontais.

A Fig. 3 ilustra a precisão, também formal, das componentes das linhas bases formadas entre as estações. Os desvios-padrão, um pouco superiores aos mostrados na Fig. 2, se devem ao fato de uma base envolver dados de duas estações, ocorrendo propagação de covariâncias. Nesta figura não se caracteriza deterioração da precisão em função do aumento do comprimento da base.

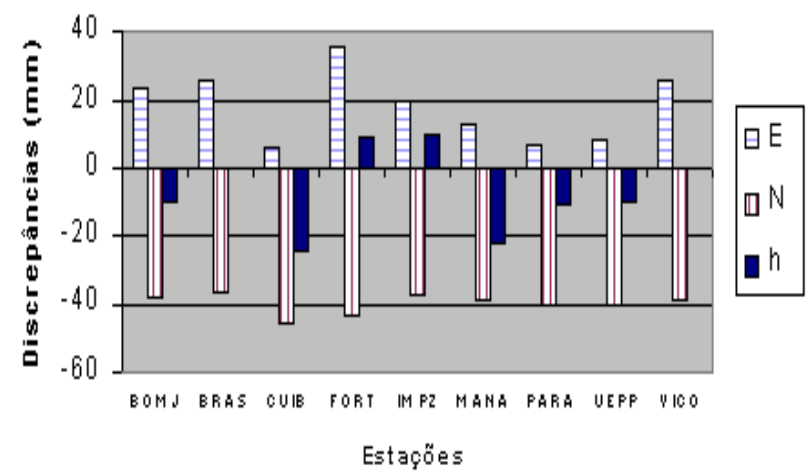

Figura 6- Discrepâncias com as coordenadas SIRGAS.

Figure 6- Discrepancies with SIRGAS coordinates.
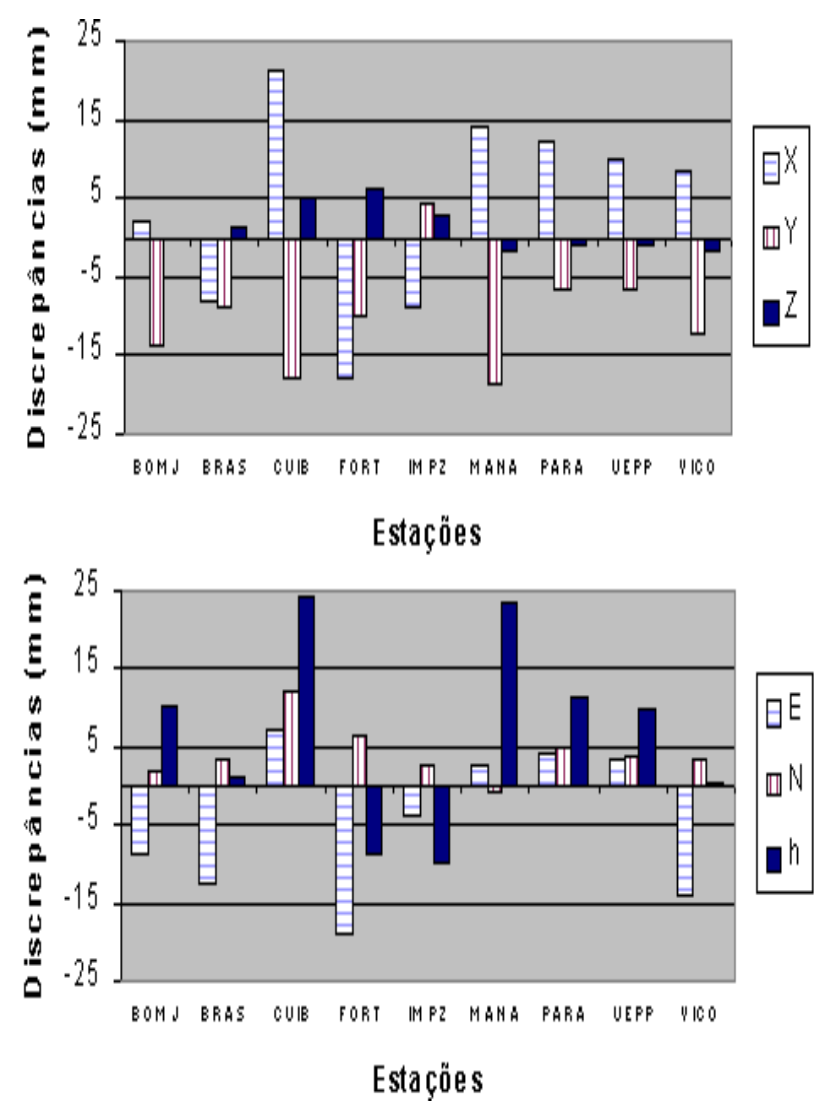

Figura 7- Discrepâncias com as coordenadas SIRGAS na época 1998,4 .

Figure 7- Discrepancies with SIRGAS coordinates at epoch 1998.4. 
A repetibilidade diária (Rep) permite uma estimativa de precisão para as coordenadas das estações ou componentes das linhas base. Trata-se do erro médio quadrático ponderado que é dado pela expressão (Blewitt, 1989):

$$
\operatorname{Re} \beta=\left(\frac{n}{n-1} \sum_{i=1}^{n} \frac{\left(R_{i}-\hat{R}\right)^{2}}{\sigma_{i}^{2}} / \sum_{i=1}^{n} \frac{1}{\sigma_{i}^{2}}\right)^{1 / 2}
$$

Nesta expressão, n é o número de dias de ocupação, $R_{i}$ e $\sigma_{i}$ são a estimativa e o erro formal (desvio-padrão) da componente da linha base para o i-ésimo dia, e $\hat{R}$ é a média ponderada da componente considerada. Os valores obtidos para a repetibilidade diária das coordenadas das estações pode ser visualizado na Fig. 4. Como esperado, a componente $\mathrm{h}$ apresenta os piores resultados, com valor mais acentuado na estação MANA. Como se trata de uma estação localizada na região amazônica, com clima totalmente adverso da maioria das demais regiões, isto pode refletir a não completa adequação da estratégia utilizada para reduzir os efeitos da refração troposférica. Mais pesquisas devem ser conduzidas neste tópico específico.

A Fig. 5 ilustra a repetibilidade em termos das componentes das linhas bases, considerando as coordenadas cartesianas $\mathrm{X}, \mathrm{Y}$ e Z e geodésicas locais E, $\mathrm{N}$ e h.

Das Figs. 2 e 4, bem como 3 e 5, pode-se observar que a repetibilidade é da ordem de 10 vezes maior que a precisão formal do ajustamento. Mas mesmo assim, a análise através da repetibilidade indica que os resultados são altamente precisos. Para as componentes geodésicas locais $\mathrm{E}$ e $\mathrm{N}$, e as três cartesianas $\mathrm{X}, \mathrm{Y}$ e Z, não há nenhum vínculo aparente entre a repetibilidade e o comprimento da linha base. A componente E proporciona repetibilidade "melhor" que $10 \mathrm{~mm}$, indicando uma boa consistência entre os diferentes dias de dados processados. Os melhores resultados, em termos de repetibilidade, estão relacionados com a componente $\mathrm{N}$, cujo valor não ultrapassa $5 \mathrm{~mm}$. Para a repetibilidade da componente $\mathrm{h}$, aparentemente, há um vínculo com o comprimento da base. Realizando uma regressão linear, obtém-se $6 m m+4 p p b$ (partes por bilhão).

No que se refere a comparação com outras técnicas e processamentos, o que há disponível para as estações incluídas neste processamento é o resultado da campanha SIRGAS (IBGE, 1997), para a época 1995,4. Uma comparação direta entre as coordenadas obtidas neste processamento (ITRF96, época 1998,4) e as coordenadas referidas ao SIRGAS apresentam as discrepâncias ilustradas na Fig. 6.

Considerando a precisão formal estimada no SIRGAS, que é de aproximadamente $4 \mathrm{~mm}$ em cada uma de suas componentes $\mathrm{X}, \mathrm{Y}$ e Z, e a do processamento deste trabalho (ver Fig. 2), as discrepâncias ilustradas na Fig. 5 não se encontram dentro de um intervalo de valores esperados. Pode-se ainda observar na Fig. 6 que as discrepâncias nas coordenadas $\mathrm{X}$ têm comportamento que pode ser considerado aleatório, mas sistemático em Y e Z. Em termos de coordenadas geodésicas locais, as componentes $\mathrm{E}$ e $\mathrm{N}$ também apresentam comportamento sistemático, sendo que o da componente $\mathrm{N}$ é mais bem definido. Este comportamento se deve, em parte, ao aspecto temporal, já que ambas séries de coordenadas deveriam ser transformadas para uma mesma época.

Definindo como época de referência a deste processamento $(1998,4)$, o qual está referenciado ao ITRF96, e transformando as coordenadas SIRGAS da época 1995,4 para 1998,4, usando o modelo de placas tectônicas NNR NUVELA-1 (McCarthy, 1996), as novas discrepâncias são apresentadas na Fig. 7.

Pode-se observar que as discrepâncias na componente $Z$, para o caso ilustrado em coordenadas cartesianas X, Y, Z, e em N, para a ilustração em coordenadas $\mathrm{E}, \mathrm{N}$ e h estão aproximadamente na ordem de sua própria precisão. O mesmo não ocorre com as demais componentes, para três estações: CUIB, FORT e MANA. No entanto, como as estações utilizadas para conectar o SIRGAS ao ITRF94 estão dentre aquelas que apresentam precisão de até $3 \mathrm{~cm}$ (Boucher et al., 1996), discrepâncias tais como as mostradas na Fig. 7 são esperadas.

\section{COMENTÁRIOS FINAIS E CONCLUSÕES}

Neste artigo apresentou-se as três possibilidades de aplicações do posicionamento por ponto utilizando o GPS. Elas podem proporcionar precisão que varia de $100 \mathrm{~m}$ a poucos milímetros. Neste trabalho foi realizado um experimento em que se aplicou o posicionamento por ponto de alta precisão, utilizando o programa GOA-II.

Os resultados foram analisados em termos de precisão formal, repetibilidade e comparação com outros resultados. A precisão formal mostrou resultados da ordem de 10 vezes melhor que a 
repetibilidade diária. Comparação com os resultados obtidos na campanha SIRGAS, utilizando o modelo de placas tectônicas NNR NUVELA-1 para atualizar as coordenadas para a época do experimento relatado neste trabalho, mostrou compatibilidade da ordem de 2,3 cm na componente $\mathrm{h}$ de duas estações (CUIB e MANA), e de $1,7 \mathrm{~cm}$ na componente E de outra estação (FORT). Tratam-se de valores relativamente elevados se comparados com a precisão dos resultados apresentados. Mas ao se considerar que as estações utilizadas para conectar o SIRGAS ao ITRF94 apresentam precisão que pode chegar a $3 \mathrm{~cm}$, tais resultados são pertinentes.

Considerando a relativa simplicidade do método, em conjunto com a precisão e acuracidade alcançados, além ainda da redução do custo computacional em relação ao processamento de redes, pode-se concluir que se trata de uma poderosa ferramenta para aplicações em atividades exigindo alta precisão em termos de posicionamento, tal como em Geodinâmica.

\section{AGRADECIMENTOS}

O suporte financeiro para a realização deste trabalho foi garantido pela FAPESP - Fundação de Amparo a Pesquisa no Estado de São Paulo dentro do programa Jovem Pesquisador em Centros Emergentes, processo 95/08775-1, e CNPq, através de Projeto Integrado de Pesquisa, Processo 523448/95-3.

\section{REFERÊNCIAS}

BIERMAN, G. J. - 1977- Factorization Method for Discrete Sequential Estimation. Academic, San Diego, $233 \mathrm{pp}$.

BLEWITT, G. - 1989- Carrier Phase Ambiguity Resolution for the Global Positioning System Applied to Geodetic Baselines up to $2000 \mathrm{~km}$. Journal of Geophysical Research, 94(B8): 10.187-10.203

BOCK, Y - 1996 - Reference System. In: KLEUSBER, A. \& TEUNISSEN, P. J .G., GPS For Geodesy. Berlin, Spring Verlag, 3-36

BOUCHER, C., ALTAMINI, Z., FEISSEL, M., \& SILLARD, P. - 1996 - Results and Analysis of the ITRF94. IERS Technical Note 20. Centrau Bureau of IERS, Observatoire de Paris, Paris, France.

BOUCHER, C., ALTAMINI, Z. \& SILLARD, P. -1997The ITRF96 Realization of the International Terrestrial Reference System. In Advances in Positioning and Reference Frames, IAG Symposia, Vol. 188, Springer, p. 57-58.

CAMARGO, P. O. -1999- Modelo Regional da Ionosfera para uso em posicionamento com receptores GPS de uma freqüência. Seminário de qualificação do curso de pós-graduação em Ciências Geodésicas da UFPR, $108 \mathrm{pp}$.

FORTES, L. P. S. -1997- Operacionalização da Rede Brasileira de Monitoramento Contínuo do Sistema GPS (RBMC). Tese de Mestrado, IME, $152 \mathrm{p}$.

GEMAEL, C. -1994- Introdução ao Ajustamento de Observações: Aplicações Geodésicas. Curitiba, PR., Editora da UFPR, 319pp.

GURTNER, W. -1994- RINEX: The receiver-Independent Exchange Format. GPS World, July, 48-52.

HÉROUX, P. \& KOUBA, J. -1995- GPS Precise Point Positioning with a Difference. http:// www.geod.emr.ca/docs/PDF, 12pp.

IBGE - INSTITUTO BRASILEIRO DE GEOGRAFIA \& ESTATÍSTICA -1997- SIRGAS Relatório Final - Grupos de Trabalho I e II. Departamento de Geodésia, Rio de Janeiro, 99pp.

LEICK, A. -1995- GPS Satellite Surveying. Wiley, 560 pp.

McCARTHY, D. D. -1996- IERS Conventions (1996). IERS Technical Note 21, Central Bureau of IERSObservatoire de Paris, 95pp.

MONICO, J. F. G. -1998- Posicionamento Pelo NAVSTAR-GPS: Descrição, Fundamentos e Aplicações. Publicação Interna, Departamento de Cartografia, $182 \mathrm{pp}$.

SILVA, N C. C. $\mathbf{- 1 9 9 8 ~ - ~ A n a ́ l i s e ~ d o ~ E f e i t o ~ d o s ~ M o d e l o s ~}$ de Refração Troposférica no Posicionamento Geodésico Usando Dados da RBMC. Tese de Mestrado, IME, 137 pp.

WEBB, F. H. \& ZUMBERGE, J. F. -1997- An Introduction to GIPSY/OASIS-II. Jet Propulsion Laboratory, JPL D-11088, Pasadena CA.

ZUMBERGE, J. F. \& BERTIGER, W. I. -1996- Ephemeris and Clock Navigation Message Accuracy. In Global Positioning System - Theory and Applications, Vol. I, Institute of Aeronautics and astronautics, Inc., Washington D.C., p.585-599

ZUMBERGE, J. F., HEFLIN, M. B., JEFFERSON, D. C., WATKINS, M. M., \& WEBB, F. H. - 1997a Precise Point Positioning for the efficient and robust analysis of GPS data from large networks. Journal of Geophysical Research, 102 (B3): 5005-5017.

ZUMBERGE, J. F., WATKINS, M. M. \& WEBB, F. H. - 1997b-Characteristics and Applications of Precise GPS Clock Solutions Every 30 Seconds. Journal of the Institute of Navigation, 44(4): 449-456.

Manuscript submitted April 30, 1999 Revised version accepted October 3, 1999 


\section{NOTA SOBRE O AUTOR}

João Francisco Galera Monico

Engenheiro Cartógrafo pela Universidade

Estadual Paulista Julio de Mesquita Filho (UNESP) em

1982, Mestrado em Ciências Geodésicas pela

Universidade Federal do Paraná em 1988 e Doutorado

em Engenharia de Levantamentos e Geodésia Espacial

pelo Instituto de Engenharia de Levantamentos e
Geodésia Espacial da Universidade de Nottingham, UK, em 1995. Desenvolve projeto de pesquisa dentro do Programa Jovem Pesquisador da FAPESP e no CNPq. As áreas de interesse são: Posicionamento Geodésico de Alta Precisão, Controle de qualidade em Geodésia, Aplicações não convencionais do GPS, Integração GLONASS e GPS.

\section{PUBLICAÇÃO DE INFORMAÇÕES INSTITUCIONAIS}

Para a publicação de informações sobre atividades de Geofísica em sua instituição, na seção ANÁLISES/RELATÓRIOS da Revista Brasiliera de Geofísica, favor submenter cópia do artigo em papel e em disquete, contendo títulos e resumos em português ou espanhol, e em inglês. $O$ artigo não poderá ultrapassar quatro páginas da Revista, incluindo ilustrações e referências.

\section{PUBLICAÇÃO DE TESES E DISSERTAÇÕES}

Para a publicação de Teses e Dissertações na Revista Brasileira de Geofísica, favor enviar uma cópia em papel e em disquete, com os títulos e resumos em português ou espanhol, e em inglês, ambos contendo um máximo de 500 palavras.

Incluir nome do autor(a), do orientador(a), departamento e universidade, título obtido, e data da defesa. Não incluir referências ou ilustrações. 